\title{
Evaluation of Clinical Outcomes of Prostatic Urethral Lift for Benign Prostatic Hyperplasia: An Asian Population Study
}

\author{
Jeong Ho Kim (iD, Ki Soo Lee ${ }^{2}$ (i), Tae Hyo Kim² (i) \\ ${ }^{1}$ Department of Urology, Dongnam Institute of Radiological and Medical Sciences, Cancer Center, ${ }^{2}$ Department of Urology, Dong-A \\ University College of Medicine, Busan, Korea
}

Purpose: This study aimed to assess the clinical outcomes of prostatic urethral lift in Korean patients with bothersome lower urinary tract symptoms due to benign prostatic hyperplasia (BPH).

Materials and Methods: Thirty-two men with symptomatic BPH were consecutively treated in a tertiary care center in Korea. To be included in the present analysis, patients had to meet the following criteria: age $\geq 50$ years, International Prostatic Symptom Score (IPSS) $>12$, and prostate volume between $30 \mathrm{~mL}$ and $80 \mathrm{~mL}$. Patients were evaluated up to a median followup period of 1 year post-procedure. The primary outcomes included symptom relief, improvement of quality of life (QOL), and preservation of sexual function.

Results: All procedures were finished with a mean of 2.2 implants without any serious complication. The numbers of patients diagnosed with diabetes mellitus, hypertension, and ischemic heart disease were $16(50.0 \%), 24(75.0 \%)$, and $9(28.1 \%)$, respectively. Patients experienced symptom relief by 1 week that was sustained for 12 months. The mean IPSS, QOL, and maximum flow rate improved to $43 \%, 70 \%$, and $25 \%$ by 1 week, and to $41 \%, 60 \%$, and $32 \%$ by 12 months ( $<<0.001$ ), respectively. There were no occurrences of early urge incontinence, retrograde ejaculation, or erectile dysfunction. Patient-reported adverse events were mild and transient.

Conclusions: Prostatic urethral lift is a safe and effective treatment for BPH in the Asian population. This treatment is minimally invasive, can be performed under local anesthesia, and may be an appropriate method for fragile patients.

Keywords: Benign prostatic hyperplasia; Lower urinary tract symptoms; Minimally invasive surgical procedures; Prostatic urethral lift; Urolift

This is an Open Access article distributed under the terms of the Creative Commons Attribution Non-Commercial License (http://creativecommons.org/licenses/by-nc/4.0) which permits unrestricted non-commercial use, distribution, and reproduction in any medium, provided the original work is properly cited.

\section{INTRODUCTION}

An aged society is a country that has more than $14 \%$ of its population aged 65 or older. South Korea became an aging society in 2000 and has remained an aged so- ciety through the years since 2000 . The trend of an increasing population of elderly individuals is occurring not only in Korea but also in other developed countries and nations worldwide. As the population ages, it is associated with many health problems, such as urinary

Received: Jan 28, 2019 Revised: Jun 18, 2019 Accepted: Jun 24, 2019 Published online Jul 30, 2019

Correspondence to: Tae Hyo Kim (iD https://orcid.org/0000-0002-5994-7878

Department of Urology, Dong-A University Hospital, 26 Daesingongwon-ro, Seo-gu, Busan 49201, Korea.

Tel: +82-51-240-2727, Fax: +82-51-253-0591, E-mail: dockim0927@hanmail.net 
tract system issues.

According to epidemiologic data in Korea, the prevalence of lower urinary tract symptoms (LUTS) increases from $78 \%$ among men in their 40 s to over $90 \%$ among men aged $\geq 60$ years [1]. LUTS usually resulting from progressive enlargement of the prostate gland considerably impair the quality of life (QOL) of older men. Benign prostatic hyperplasia (BPH) is a common disease in older men. The management of bothersome $\mathrm{BPH}$ includes watchful waiting, pharmacotherapy, and surgery. Typically, the first course of treatment is watchful waiting [2]. Drugs such as $\alpha$-blockers, $5-\alpha$ reductase inhibitors, and anticholinergics can be used for pharmacotherapy. Although pharmacotherapy is effective and safe for treating LUTS, $25 \%$ to $70 \%$ of patients with $\mathrm{BPH}$ are non-compliant or discontinue medications altogether because of insufficient relief or bothersome side effects [3,4]. The most common surgical approach is transurethral resection of the prostate (TURP), which uses transurethral electrocautery to remove prostate tissue during irrigation [5]. TURP can improve voiding symptoms dramatically, and it is the most widely used procedure for prostate gland removal [6]. However, TURP is correlated with a $20 \%$ rate of perioperative and long-term complications that include early urge incontinence (30\%-40\%), late iatrogenic urinary incontinence (1\%-3\%), bleeding requiring transfusions (2.9\%), ejaculatory dysfunction (65\%), urethral stricture (2.2\%-9.8\%), erectile dysfunction (10\%), and transurethral resection syndrome (1.4\%) [7-9]. In addition, elderly patients usually have some comorbidities that require complex medical regimens that may further complicate perioperative management [10]. Therefore, new treatments that are more minimally invasive and more effective are needed to manage patients with LUTS.

Prostatic urethral lift (PUL) is a minimally invasive surgery that uses small implants under cystoscopic guidance. These permanent transprostatic implants serve to retract the obstructing lateral lobes and make an open, continuous voiding channel through the prostate fossa, from the verumontanum up to the bladder neck. PUL has been demonstrated to provide significant symptom improve with low morbidity, including preservation of sexual function [7,11,12]. This procedure can be performed under local anesthesia because it does not involve resection, ablation, or other thermal injury to the prostate. In the era of an increasingly ag- ing population, a surgery that can be performed under local anesthesia is beneficial. Several studies for PUL have provided clinicians and patients with promising results, but no studies have examined the therapeutic effect of this procedure in Asian patients. Therefore, this study aimed to evaluate the efficacy and tolerability of PUL in Korean patients with BPH.

\section{MATERIALS AND METHODS}

\section{Ethical statement}

This study was approved by the Institution Review Board of Dong-A University Hospital and conducted according to the ethical standard laid down by the 1964 declaration of Helsinki and its later amendments. Written informed consent was obtained from all study participants.

\section{Study design and patients}

We performed a retrospective study of patients with BPH treated with PUL in a tertiary care center in Korea between 2015 and 2017. The inclusion criteria were as follows: age $\geq 50$ years, International Prostatic Symptom Score (IPSS) $>12$, and prostate volume between $30 \mathrm{~mL}$ and $80 \mathrm{~mL}$. Baseline demographics and clinical data were collected.

\section{Surgical procedure, follow-up, and outcomes}

The PUL procedure is conducted by installing small implants (Urolift ${ }^{\circledR}$ System; NeoTract, Pleasanton, CA, USA) transurethrally under cystoscopic guidance to compress the enlarged prostatic lateral lobes and reduce obstruction [13]. The determination of the number of implants is dependent on the adenoma size and configuration, and typically, two implants are used to create a continuous anterior voiding channel. Patients were followed-up during visits at 1 week, 1 month, 6 months, and 1 year after the procedure. We followed the surgical technique introduced previously [13]. The IPSS was to check for subjective improvement of urinary symptoms. Uroflowmetry was performed to measure objective urinary state. The International Index of Erectile Function 5 (IEF5) scale was used to check for reduced erectile functions. IPSS, IIEF5, and Uroflowmetry were administered for all patients at each followup. Primary outcome measures included the IPSS, IIEF, and maximum urine flow rate (Qmax). 


\section{Statistical analysis}

The changes in baseline characteristics and outcomes at multiple time points were analyzed using the paired Student's t-test or Wilcoxon's signed-rank test depending on the distribution of the paired data. A general estimating equation model was fitted to each study output parameter. The change from baseline was the dependent variable, and the scores at baseline and follow-up visit were used as independent variables. An exchangeable correlation structure and identity link were used. Continuous variables are expressed as a mean \pm standard deviation, whereas categorical variables are demonstrated as a number (percentage). All statistical analyses were performed using IBM SPSS ver. 20 software (IBM Corp., Armonk, NY, USA), and a $\mathrm{p}<0.05$ indicated statistical significance.

\section{RESULTS}

Thirty-two patients were included in this study. The patients' preoperative clinical characteristics are summarized in Table 1. An average of 2.2 implants were placed, with two implants being the most frequent (87.5\%). All procedures were performed under local anesthesia using cold lidocaine with a sedative. Significant improvements in the IPSS, IPSS QOL, and Qmax were observed after the procedure through the 1-year follow-up (Table 2). The mean total reduction in the IPSS after PUL was $43 \%$ at 1 week and that was main-

Table 1. Baseline patient characteristics $(n=32)$

\begin{tabular}{lc}
\hline \multicolumn{1}{c}{ Variable } & Value \\
\hline Age $(\mathrm{y})$ & $67 \pm 7$ \\
Prostate volume $(\mathrm{mL})$ & $50 \pm 7$ \\
DM & $16(50.0)$ \\
Hypertension & $24(75.0)$ \\
Ischemic heart disease & $9(28.1)$ \\
IPSS & \\
Total score & $19.3 \pm 2.4$ \\
Storage subscore & $7.8 \pm 1.8$ \\
Voiding subscore & $11.5 \pm 2.0$ \\
QOL & $4.4 \pm 0.6$ \\
IIEF5 & $18.8 \pm 4.7$ \\
Qmax (mL/s) & $12.1 \pm 2.4$ \\
\hline
\end{tabular}

Values are presented as mean \pm standard deviation or number (\%). DM: diabetes mellitus, IPSS: International Prostate Symptom Score, QOL: quality of life, IIEF: International Index of Erectile Function, Qmax: maximum urinary flow rate. tained throughout the follow-up duration. A significant decrease in the IPSS QOL to 30\% of the preoperative level was observed in the first 1 week after PUL. Qmax showed rapid improvement, increasing from $12.1 \mathrm{~mL} / \mathrm{s}$ at baseline to $16 \mathrm{~mL} / \mathrm{s}$ at 1 week after PUL, and maintaining to $15.1 \mathrm{~mL} / \mathrm{s}$ and $15.3 \mathrm{~mL} / \mathrm{s}$ at 6 and 12 months after PUL, respectively. Patients' symptoms were quickly improved after PUL, and clinical outcomes nearly peaked within a week (Fig. 1-3). The continence function was maintained in all patients throughout the follow-up duration and did not change significantly at any time point. There was no significant degradation in the mean erectile function (as measured by IIEF5) over the course of 1 year (Fig. 4). Perioperative adverse

Table 2. Clinical outcomes after prostatic urethral lift

\begin{tabular}{|c|c|c|c|c|}
\hline Variable & 1 week & 1 month & 6 months & 12 months \\
\hline \multicolumn{5}{|l|}{ IPSS } \\
\hline Baseline $^{a}$ & $19.3 \pm 2.4$ & $19.3 \pm 2.4$ & $19.3 \pm 2.4$ & $19.3 \pm 2.4$ \\
\hline Follow-up ${ }^{a}$ & $10.9 \pm 1.9$ & $10.8 \pm 1.9$ & $10.9 \pm 1.8$ & $11.2 \pm 1.7$ \\
\hline Change & -8.4 & -8.4 & -8.4 & -8 \\
\hline$\%$ change & -43 & -43 & -43 & -41 \\
\hline $95 \% \mathrm{Cl}$ & -47 to -40 & -47 to -39 & -47 to -39 & -45 to -37 \\
\hline$p$-value & $<0.001$ & $<0.001$ & $<0.001$ & $<0.001$ \\
\hline \multicolumn{5}{|l|}{$\mathrm{QOL}$} \\
\hline Baseline $^{a}$ & $4.4 \pm 0.6$ & $4.4 \pm 0.6$ & $4.4 \pm 0.6$ & $4.4 \pm 0.6$ \\
\hline Follow-up ${ }^{a}$ & $1.3 \pm 0.5$ & $1.4 \pm 0.6$ & $1.7 \pm 0.6$ & $1.7 \pm 0.6$ \\
\hline Change & -3.1 & -3 & -2.7 & -2.7 \\
\hline$\%$ change & -70 & -69 & -60 & -60 \\
\hline $95 \% \mathrm{Cl}$ & -74 to -65 & -73 to -64 & -65 to -55 & -65 to -55 \\
\hline $\mathrm{p}$-value & $<0.001$ & $<0.001$ & $<0.001$ & $<0.001$ \\
\hline \multicolumn{5}{|l|}{ IIEF5 } \\
\hline Baseline $^{a}$ & $18.8 \pm 4.7$ & $18.8 \pm 4.7$ & $18.8 \pm 4.7$ & $18.8 \pm 4.7$ \\
\hline Follow-up ${ }^{a}$ & $17.9 \pm 4.7$ & $17.3 \pm 4.6$ & $17.7 \pm 4.3$ & $17.9 \pm 4.5$ \\
\hline Change & -0.9 & -1.5 & -1.1 & -0.9 \\
\hline$\%$ change & -7.1 & -6.6 & -3.2 & -3.5 \\
\hline $95 \% \mathrm{Cl}$ & -14 to -0.1 & -13 to -0.3 & -12.3 to 5.9 & -9.9 to 2.9 \\
\hline p-value & 0.159 & 0.019 & 0.041 & 0.129 \\
\hline \multicolumn{5}{|l|}{ Qmax $(\mathrm{mL} / \mathrm{s})$} \\
\hline Baseline $^{a}$ & $12.1 \pm 2.4$ & $12.1 \pm 2.4$ & $12.1 \pm 2.4$ & $12.1 \pm 2.4$ \\
\hline Follow-up ${ }^{a}$ & $16 \pm 1.3$ & $15.6 \pm 1.6$ & $15.1 \pm 1.4$ & $15.3 \pm 1.4$ \\
\hline Change & 3.9 & 3.5 & 3.1 & 3.3 \\
\hline$\%$ change & 25 & 34 & 30 & 32 \\
\hline $95 \% \mathrm{Cl}$ & 20 to 30 & 24 to 44 & 21 to 40 & 22 to 42 \\
\hline $\mathrm{p}$-value & $<0.001$ & $<0.001$ & $<0.001$ & $<0.001$ \\
\hline
\end{tabular}

IPSS: International Prostate Symptom Score, Cl: confidence interval, QOL: quality of life, IIEF: International Index of Erectile Function, Qmax: maximum urinary flow rate.

${ }^{\mathrm{a}}$ Mean \pm standard deviation. 


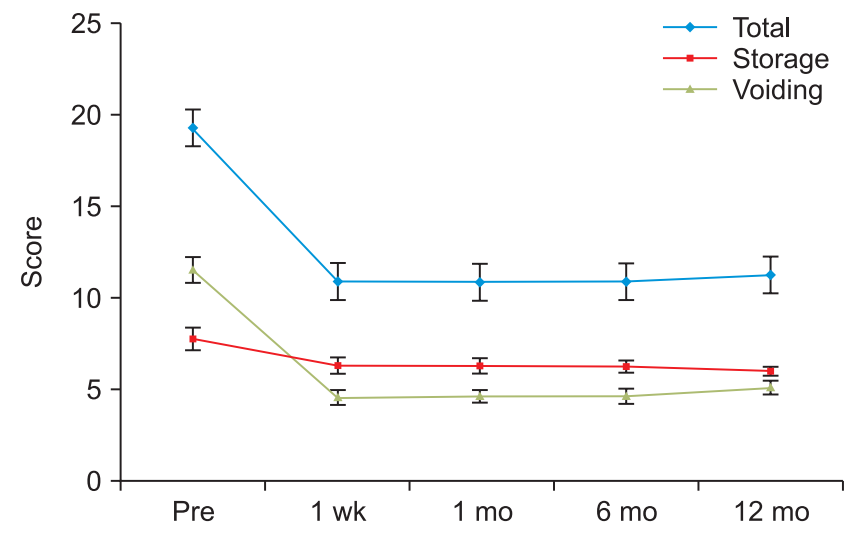

Fig. 1. Change of the International Prostatic Symptom Score from baseline over time. Values are represented as the mean with $95 \%$ confidence interval. Pre: preoperatively.

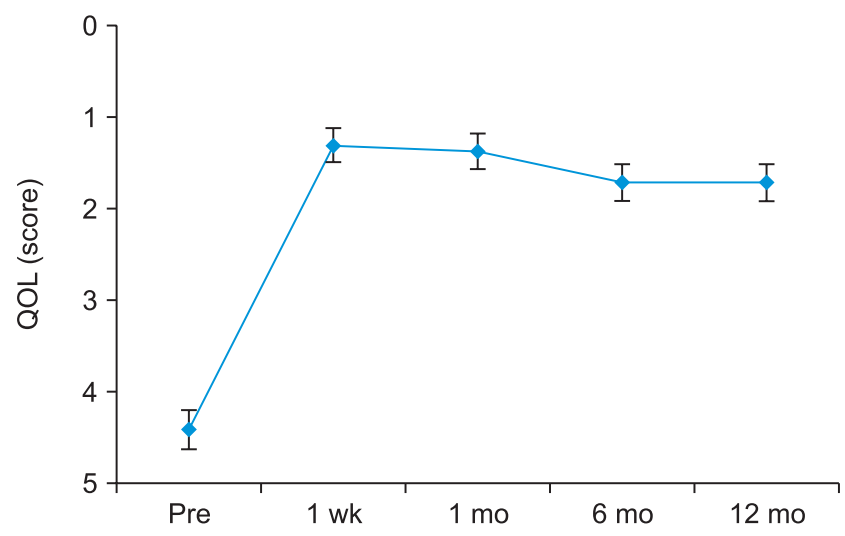

Fig. 2. Sequential changes in the QOL score after PUL. Values are represented as the mean with $95 \%$ confidence interval. Pre: preoperatively, QOL: quality of life, PUL: prostatic urethral lift.

events were typically mild and transient, with the most frequent being hematuria. There was no reported incidence of new retrograde ejaculation or erectile dysfunction after PUL. After 1 year, follow-up cystoscopy demonstrated that no implant was improperly deployed within the prostatic urethra or showed signs of encrustation. Thus, no patient experienced removal of implants due to misdeployment. Over the 1-year followup, no patients required surgical retreatment for recurrence of LUTS. A total of 10 patients were diagnosed with underactive bladder (UAB). UAB was defined as cystoscopic findings of severe trabeculation, along with post-void residual urine (PVR) $\geq 300 \mathrm{~mL}$ and Qmax $<10 \mathrm{~mL} / \mathrm{s}$. A separate analysis of patient data showed significant improvement in IPSS, Qmax, and PVR to $37.3 \%, 44.7 \%$, and $47.6 \%$, respectively, after 12 months.

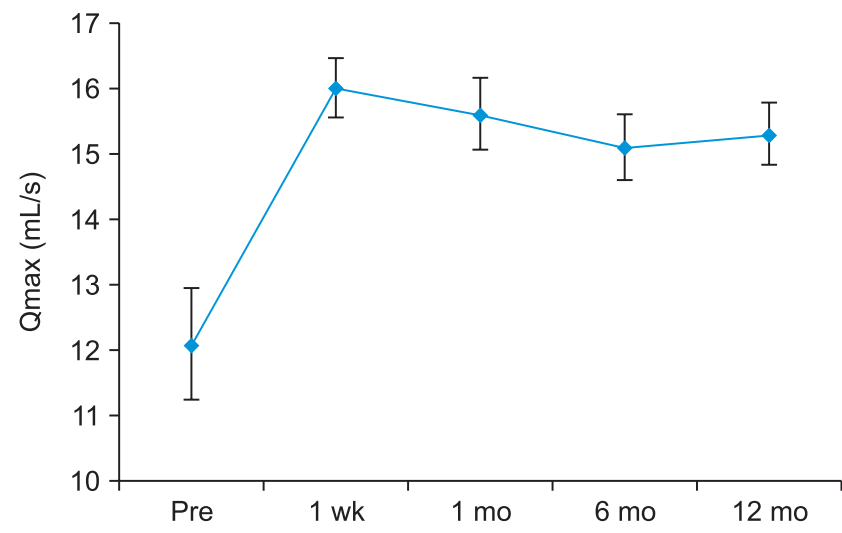

Fig. 3. Sequential changes in the Qmax after PUL. Values are represented as the mean with $95 \%$ confidence interval. Pre: preoperatively, Qmax: maximum flow rate, PUL: prostatic urethral lift.

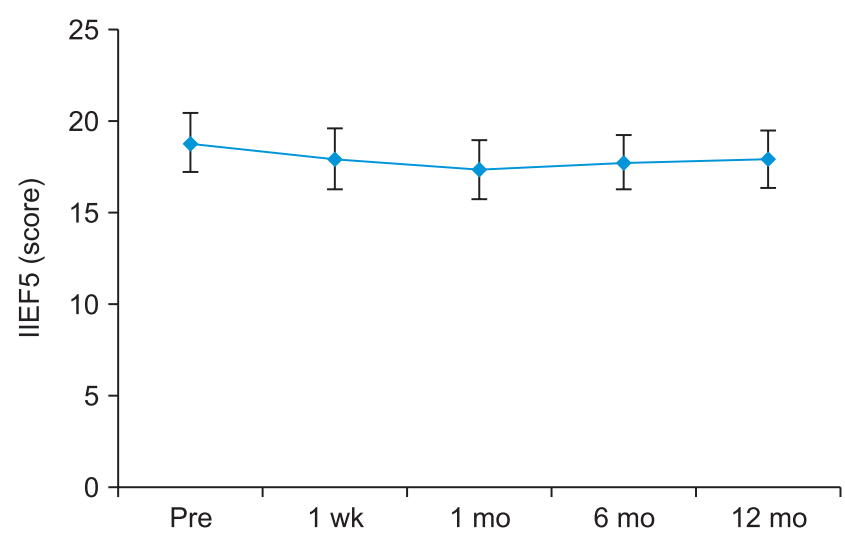

Fig. 4. Sequential changes in the IIEF5 score after PUL. Values are represented as the mean with $95 \%$ confidence interval. Pre: preoperatively, IIEF: International Index of Erectile Function, PUL: prostatic urethral lift.

\section{DISCUSSION}

According to a cross-sectional community-based survey in Korean men, the overall prevalence of $\mathrm{BPH}$ and moderate to severe LUTS were $21.0 \%$ and $37.6 \%$, respectively [14]. The incidence of moderate to severe LUTS significantly increases with age [14]. LUTS may degrade the QOL in men; therefore, effective treatment for relieving their symptoms is needed. The results of this study showed that PUL can offer durable efficacy as treatment for LUTS without severe adverse events. The IPSS decreased from baseline by $43 \%$ at 1 week and by $41 \%$ at 1 year in the evaluated patients. Outcomes were maintained up to the followup assessment at 12 months. Therefore, PUL can improve subjective symptoms and QOL in patients with BPH/LUTS. These results are consistent with those of 
previous studies of PUL [7,11,12,15,16]. Most previous studies were performed in Western countries, whereas the present study was conducted in an Asian country. Therefore, we think that PUL is a valid alternative to minimally invasive therapy in patients with $\mathrm{BPH}$, including Asian patients.

The effects of PUL in relieving symptoms were rapid. In this study, reduction in the IPSS and QOL score reached near the maximum percentage of improvements within a week. The remarkable effects of $\alpha$-blockers on urinary symptoms are seen within 1 week of starting the medication, but it takes a few weeks until they reach their maximal level of efficacy [17,18]. In a comparative study of PUL and TURP, PUL improved symptoms more rapidly than TURP $[7,16]$. An Australian multicenter study reported similar results. Chin et al [15] studied PUL in 64 patients with $\mathrm{BPH}$, and the IPSS decreased by $42 \%$ at 2 weeks, $49 \%$ at 6 months, and $42 \%$ at 2 years in the evaluated patients. This advantage of PUL might help improve patients' QOL.

Patients older than 70 years of age are more likely to have multiple comorbidities, reduced renal, cardiac, and pulmonary function, and physical and cognitive impairments [10]. These factors affect older patients to an increased risk for perioperative complications and mortality. For this reason, the surgery can be performed under local anesthesia with or without sedation and would be a useful tool in the management of older patients with BPH. In this study, all procedures were performed under local anesthesia using cold lidocaine with sedative, and no patient complained of pain or discomfort. Therefore, we think that PUL may be an attractive treatment option for $\mathrm{BPH}$ in the aged society.

Objective benefits were also observed in the present study, such as improvements of up to $3.9 \mathrm{~mL} / \mathrm{s}$ in the Qmax during the 1-month and 12-month follow-ups. These outcomes cannot be directly compared to those of current medical and minimally invasive therapy, as they are non-inferior. TURP has shown superior outcomes in the improvement of Qmax in a prospective, randomized comparative study [7,16]. Therefore, proper patient selection is very important in managing patient expectations. Cavitation surgery may be more appropriate for patients with severe LUTS and a huge prostate, but PUL would be one of the alternative treatment options for patients with relatively mild symptoms.

Treatment-related adverse events were mild and transient. A favorable safety profile is known as one of the main benefits of PUL. The most common adverse events correlated with PUL include dysuria, hematuria, and pelvic pain [19-23]. These adverse events are typically resolved within the first week. No serious adverse events were reported in previous reports $[7,11,12,15,16]$. Similar results were also observed in this study. Hematuria was observed most frequently in the present study, which resolved with supportive care within 2 weeks. Continence function was maintained during the follow-up period. Deterioration of continence was significantly correlated with a clinically important decrease in health-related QOL. Studies of TURP reported that early urge incontinence occurs in up to $30 \%$ to $40 \%$ of patients [5]. Gratzke et al [7] reported that the PUL group was found to have stable incontinence severity index scores, but patients in the TURP group experienced a significant decrease in continence at 2 weeks and 3 months. Overall, PUL has a benefit without compromising urinary continence. Because PUL does not affect the integrity of the bladder neck, antegrade ejaculation is maintained. Likewise, no patients complained of ejaculation dysfunction in our study. In this regard, PUL has had the greatest advantage of both the preservation of continence function and improvement of the QOL.

$\mathrm{UAB}$ refers to the complicated condition associated with difficulty in expelling urine from the bladder, which is characterized by major symptoms such as prolonged urination time, hesitancy, reduced sensation on filling, and a slow stream [24]. According to Ko et al [25], UAB involves detrusor underactivity (DU) overlapping with bladder outlet obstruction (BOO), overactive bladder, and/or stress urinary incontinence. According to a report in South Korea, DU was observed in $40.2 \%$ of men with LUTS [26]. There is still some controversy on whether resolving $\mathrm{BOO}$ by surgical intervention is helpful for patients with UAB who complain of LUTS. However, some recent studies have reported that surgically treating $\mathrm{BOO}$ could be helpful in improving patient symptoms [26,27]. The present study also found improvement in objective indicators and subjective symptoms when using Urolift to treat BOO in elderly patients with UAB who had difficulties with anesthesia due to multiple comorbidities.

Limitations of this study include the small sample 
size, which may not have provided sufficient statistical power. Second, this was a retrospective study; therefore, we cannot exclude the possibility of selection bias. Third, the aim of this study was to examine the response of patients following the PUL procedure in a real clinical setting. Thus, this study's findings cannot be objectively compared to those of other therapies.

\section{CONCLUSIONS}

PUL is a safe and effective treatment for BPH in the Asian population. Subjective symptoms and objective variables rapidly improved after PUL without severe adverse events. This treatment is minimally invasive, it can be performed under local anesthesia, and it may be an appropriate method for fragile patients.

\section{Conflicts of Interest}

The authors have no potential conflicts of interest to disclose.

\section{Author Contribution}

Conceptualization: all authors. Data curation: KSL, THK. Formal analysis: JHK. Project administration: THK. Supervision: THK. Validation: KSL. Writing-original draft: JHK. Writingreview \& editing: THK.

\section{Data Sharing Statement}

The data analyzed for this study have been deposited in HARVARD Dataverse and are available at https://doi. org/10.7910/DVN/KGEUGY.

\section{REFERENCES}

1. Kim TH, Han DH, Lee KS. The prevalence of lower urinary tract symptoms in korean men aged 40 years or older: a population-based survey. Int Neurourol J 2014;18:126-32.

2. Thorpe A, Neal D. Benign prostatic hyperplasia. Lancet 2003;361:1359-67.

3. Cindolo L, Pirozzi L, Fanizza C, Romero M, Tubaro A, Autorino $\mathrm{R}$, et al. Drug adherence and clinical outcomes for patients under pharmacological therapy for lower urinary tract symptoms related to benign prostatic hyperplasia: population-based cohort study. Eur Urol 2015;68:418-25.

4. Verhamme KM, Dieleman JP, Bleumink GS, Bosch JL, Stricker BH, Sturkenboom MC. Treatment strategies, patterns of drug use and treatment discontinuation in men with LUTS suggestive of benign prostatic hyperplasia: the Triumph project. Eur Urol 2003;44:539-45.

5. Rassweiler J, Teber D, Kuntz R, Hofmann R. Complications of transurethral resection of the prostate (TURP): incidence, management, and prevention. Eur Urol 2006;50:969-79; discussion 980 .

6. Oelke M, Bachmann A, Descazeaud A, Emberton M, Gravas S, Michel MC, et al.; European Association of Urology. EAU guidelines on the treatment and follow-up of non-neurogenic male lower urinary tract symptoms including benign prostatic obstruction. Eur Urol 2013;64:118-40.

7. Gratzke C, Barber N, Speakman MJ, Berges R, Wetterauer U, Greene D, et al. Prostatic urethral lift vs transurethral resection of the prostate: 2-year results of the BPH6 prospective, multicentre, randomized study. BJU Int 2017;119:767-75.

8. Miano R, De Nunzio C, Asimakopoulos AD, Germani S, Tubaro A. Treatment options for benign prostatic hyperplasia in older men. Med Sci Monit 2008;14:RA94-102.

9. Reich O, Gratzke C, Bachmann A, Seitz M, Schlenker B, Hermanek P, et al.; Urology Section of the Bavarian Working Group for Quality Assurance. Morbidity, mortality and early outcome of transurethral resection of the prostate: a prospective multicenter evaluation of 10,654 patients. J Urol 2008;180:246-9.

10. Baquero GA, Rich MW. Perioperative care in older adults. J Geriatr Cardiol 2015;12:465-9.

11. Cantwell AL, Bogache WK, Richardson SF, Tutrone RF, Barkin J, Fagelson JE, et al. Multicentre prospective crossover study of the 'prostatic urethral lift' for the treatment of lower urinary tract symptoms secondary to benign prostatic hyperplasia. BJU Int 2014;113:615-22.

12. Roehrborn CG, Barkin J, Gange SN, Shore ND, Giddens JL, Bolton DM, et al. Five year results of the prospective randomized controlled prostatic urethral L.I.F.T. study. Can J Urol 2017;24:8802-13.

13. McNicholas TA, Woo HH, Chin PT, Bolton D, Fernández Arjona $\mathrm{M}$, Sievert KD, et al. Minimally invasive prostatic urethral lift: surgical technique and multinational experience. Eur Urol 2013;64:292-9.

14. Huh JS, Kim YJ, Kim SD. Prevalence of benign prostatic hyperplasia on Jeju Island: analysis from a cross-sectional community-based survey. World J Mens Health 2012;30:1317.

15. Chin PT, Bolton DM, Jack G, Rashid P, Thavaseelan J, Yu RJ, et al. Prostatic urethral lift: two-year results after treatment for lower urinary tract symptoms secondary to benign prostatic hyperplasia. Urology 2012;79:5-11. 
16. Sønksen J, Barber NJ, Speakman MJ, Berges R, Wetterauer U, Greene D, et al. Prospective, randomized, multinational study of prostatic urethral lift versus transurethral resection of the prostate: 12-month results from the BPH6 study. Eur Urol 2015;68:643-52.

17. Michel MC, Vrydag W. Alpha1-, alpha2- and beta-adrenoceptors in the urinary bladder, urethra and prostate. Br J Pharmacol 2006;147 Suppl 2:S88-119.

18. Wilt TJ, Mac Donald R, Rutks I. Tamsulosin for benign prostatic hyperplasia. Cochrane Database Syst Rev 2003;(1):CD002081.

19. Garcia C, Chin P, Rashid P, Woo HH. Prostatic urethral lift: a minimally invasive treatment for benign prostatic hyperplasia. Prostate Int 2015;3:1-5.

20. Jones P, Rai BP, Aboumarzouk O, Somani BK. UroLift: a new minimally-invasive treatment for benign prostatic hyperplasia. Ther Adv Urol 2016;8:372-6.

21. Jones P, Rajkumar GN, Rai BP, Aboumarzouk OM, Cleaveland P, Srirangam SJ, et al. Medium-term outcomes of urolift (minimum 12 months follow-up): evidence from a systematic review. Urology 2016;97:20-4.
22. Perera M, Roberts MJ, Doi SA, Bolton D. Prostatic urethral lift improves urinary symptoms and flow while preserving sexual function for men with benign prostatic hyperplasia: a systematic review and meta-analysis. Eur Urol 2015;67:70413.

23. Tsui JF, Dixon CM. Urolift: a new face of minimally invasive surgical technique for benign prostatic hyperplasia? Curr Urol Rep 2016;17:63.

24. Uren AD, Drake MJ. Definition and symptoms of underactive bladder. Investig Clin Urol 2017;58:S61-7.

25. Ko KJ, Lee CU, Lee KS. Clinical implications of underactive bladder. Investig Clin Urol 2017;58:S75-81.

26. Jeong SJ, Kim HJ, Lee YJ, Lee JK, Lee BK, Choo YM, et al. Prevalence and clinical features of detrusor underactivity among elderly with lower urinary tract symptoms: a comparison between men and women. Korean J Urol 2012;53:342-8.

27. Sokhal AK, Sinha RJ, Purkait B, Singh V. Transurethral resection of prostate in benign prostatic enlargement with underactive bladder: a retrospective outcome analysis. Urol Ann 2017;9:131-5. 